\title{
Detection of Illegal Dumping from CCTV at Recycling Centres
}

\author{
Naomi Harte, Andrew Rankin, Gary Baugh, Anil Kokaram \\ Electronic and Electrical Engineering, \\ Trinity College Dublin, Ireland. \\ nharte@tcd.ie
}

\begin{abstract}
This paper describes initial work on a framework for automatic detection of illegal dumping from CCTV footage from recycle centres. Frames are seperated into foreground and background regions using a Bayesian approach that combines global motion estimates with image based information to generate a robust segmentation. The framework hence avoids explicit modelling and tracking of objects in the scene such as cars, people or rubbish bags. A feature extraction stage with diagnostics will be presented.
\end{abstract}

\section{Introduction}

The application area of the work presented in this paper is the CCTV monitoring of Bring Banks. A Bring Bank is a collection of recycling bins for materials such as glass bottles, drinks cans and plastics, located close to large centres of population to enable people to recycle waste that is not currently collected directly from homes. They are generally unmanned and accessible 24-hours. A recent study [1] found that one in three Irish adults admitted to littering/dumping at bring banks and that $60 \%$ of people believe littering at bring banks is a problem. However, the same research found that a high proportion of people who leave recyclable materials at the banks do not see themselves as guilty of dumping. This is evidenced by the fact that $83 \%$ of people who admitted to leaving recyclables on the ground agreed that this was a real problem but only $57 \%$ thought that those who do so should be prosecuted. Illegal dumping is a significant barrier to the wider deployment of these Bring Banks and an increase in consumer recycling rates.

Local councils are amenable to the use of CCTV to address this problem. However, they generally do not have the available manpower to monitor the video. Due to the sporadic nature of the reporting of dumping, the council may be faced with days of tape to process in order to find whether the culprit was caught on camera, or indeed whether the relavant video has already been recorded over. To help automate this task and move towards automatic flagging of
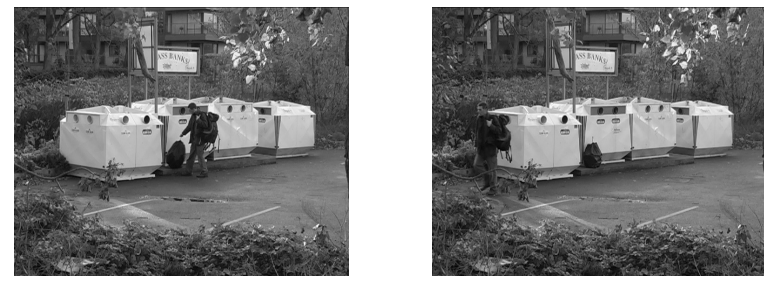

Figure 1. Frames from video of Bring Bank depicting dumping event.

suspicious events as they occur, the work presented in this paper is focussed on detecting illegal dumping in CCTV footage taken at Bring Banks to detect events such as that depicted in Figure 1. It is envisaged that the algorithm will eventually be incorporated into a video surveillance system to allow fast detection of illegal dumping at remote sites, hence enabling a more immediate response from councils and litter wardens.

\section{Framework}

Motion has been exploited as an important cue for video content analysis for a diverse range of applications from sports to surveillance. The current framework exploits a motion based video matting technique similar to [2]. This allows each frame to be divided into foreground and background. Analysis of features extracted from the resulting segmentation allow a measure of activity and change in the scene. Results presented will demonstrate how dumping events can be flagged by the system by building chains of events to be tracked and flagged as suspicious if subsequently unresolved by the system diagnostics.

\section{References}

[1] Report available on www.repak.ie, "Increased Recycling Rates Marred by Bring Bank Dumpers", 2005.

[2] Kokaram, A., Collis, B., Robinson, S., "Practical Motion Based Video Matting", IEE European Conference on Visual Media Production, pp130-136, 2005 